\title{
DESIGN AND MANUFACTURING OF THE CASSEGRAIN INTERFACE MODULE OF THE BOAO ECHELLE SPECTROGRAPH
}

\author{
Kang-Min KIM ${ }^{1,2}$, Be-Ho JAnG ${ }^{1}$, Inwoo Han ${ }^{1}$, Jeong Gyun Jang ${ }^{1}$, Hyun Chul Sung ${ }^{1}$, Moo-Young Chun ${ }^{1}$, Siek \\ HyunG $^{3}$, TAE-SEOG YoON ${ }^{2}$, AND Steven S. VOGT ${ }^{4}$ \\ ${ }^{1}$ Korea Astronomy Observatory, Daejon 305-348, Korea \\ E-mail: kmkim@boao.re.kr \\ ${ }^{2}$ Department of Astronomy and Atmospheric Sciences, Kyungpook National University, Taegu 702-701, Korea \\ ${ }^{3}$ School of Science (Astronomy), Chungbuk National University, Chongju, Chungpook 361-763, Korea \\ ${ }^{4}$ UCO/Lick Observatory, University of Califonia at Santa Cruz, Santa Cruz, CA 95064, U.S.A.
}

(Received May 30, 2002; Accepted Dec. 3, 2002)

\begin{abstract}
Cassegrain interface module (CIM) of the fiber-fed high resolution echelle spectrograph has been designed and manufactured for the $1.8 \mathrm{~m}$ reflector at the Bohynsan Optical Astronomy Observatory. We also constructed a long slit spectrograph attached to this CIM, which would replace the earlier rather inefficient medium dispersion spectrometer. We present detailed description for design and manufacturing concepts of the CIM which consists of a slit assembly, slit monitoring system, calibration lamp system and a long slit spectrograph, in order to provide how the overall system and each part are constructed. The preliminary performance test carried out so far seems to indicate a successful result.
\end{abstract}

Key words : astronomical instrumentation, fiber-fed spectrograph

\section{INTRODUCTION}

We made a fiber-fed high resolution echelle spectrograph for the $1.8 \mathrm{~m}$ reflector at the Bohyunsan Optical Astronomy Observatory (BOAO). The echelle spectrograph called "BOES" (BOao Echelle Spectrograph) is scheduled to be served to visiting astronomers from the second semester of 2003. The BOES consists of three main parts; 1 ) the CIM (Cassegrain Interface Module) part, 2) the light transmitting part, i.e. fiber sets, and 3 ) the spectrometer, i.e. a bench mounted echelle spectrograph. Design concepts of the echelle spectrograph part of the BOES including the key sciences are described in Kim et al. (2000). In this paper, we present the optical and mechanical design concepts of the CIM and the fiber sets.

The CIM, attached to the Cassegrain focus of the 1.8 $m$ reflector, has a slit assembly, a slit monitoring system and a calibration lamp system. Iodine cell (Marcy and Butler 1992) is placed at the front of the slit assembly for a precise radial velocity measurement. In addition, we newly constructed a long slit spectrograph (LSS), attached to the CIM. The high dispersion spectroscopic observations with the BOES and the medium dispersion observations with the LSS can be carried out relatively easily by switching their mode within about three minutes. Therefore one does not have to change the secondary mirror of the $1.8 \mathrm{~m}$ reflector and telescope backend instruments any longer.

The optical layout of the CIM is given in Figure 1 . The slit monitoring system is shown at the left side,

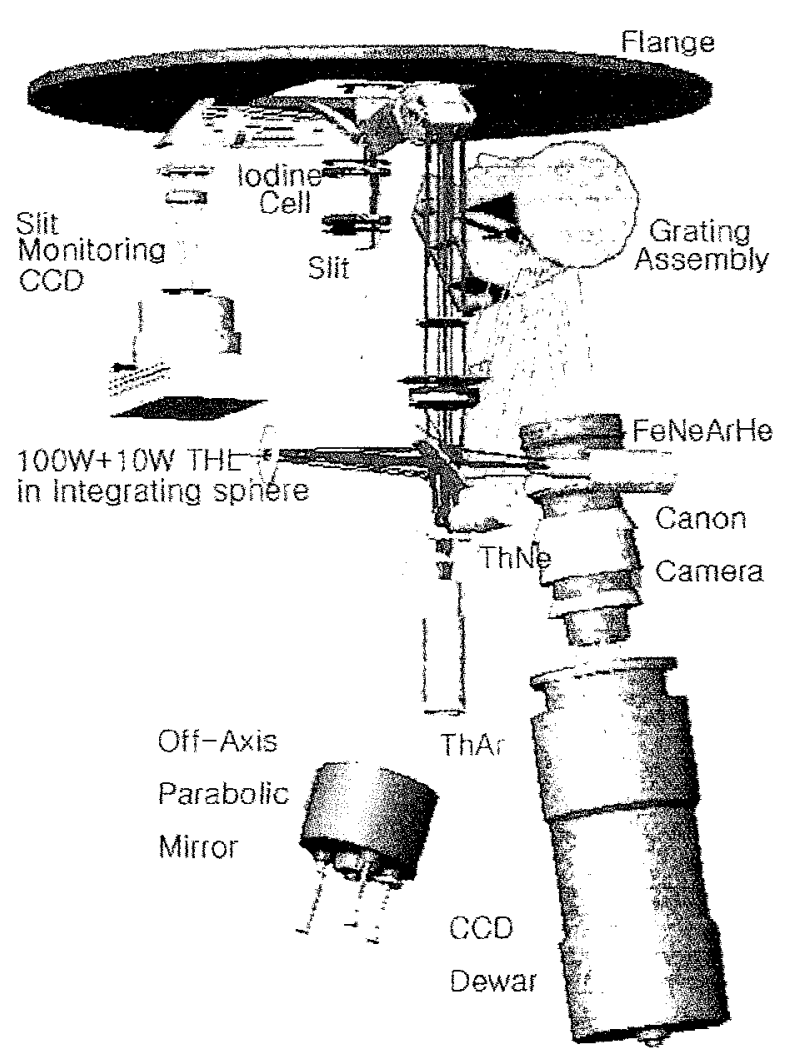

Fig. 1. - Layout of the CIM Optical Parts 
while the LSS at the right, and the calibration lamp system at the front. The back side is empty for a hand adjustment and management to the inner parts. We disposed the slit monitoring system and the LSS in opposite side from the telescope axis to make the image quality along the slit nearly the same.

Below the telescope flange, there are two mirrors one for the slit monitoring and another for the calibration light source, a iodine cell and a slit assembly. Since the space between the flange and the iodine cell is so compact, we put two mirrors in a mount on a linear translator and move the mirror mount by DC motor.

We adapted an off-axis parabolic mirror as the collimator of the LSS. It makes the CIM a long shape, which is easily affected by gravity. To minimize the bending due to gravity, we installed several ribs inside the CIM and long cylindrical type collimator housing.

We put the CIM controller at the left of the slit monitoring system to maintain the torque balance due to the gravitation. The telescope image derotator works well with the CIM without any counter balance device. The total weight of the CIM including the flange is 250 $\mathrm{kg}$.

\section{SLIT ASSEMBLY}

The BOES has a long slit for medium dispersion observations on extended sources using the LSS, and a fiber slit (fiber input) for high dispersion using the echelle spectrograph. These slits are installed on a 100 $\mathrm{mm}$ translation stage (Figure 2, the slit cover removed for viewing). Slit selection can be controlled with a stepping motor.

The long slits were made by laser drilling on a 0.2 $\mathrm{mm}$ thick stainless steel. The physical length of the long slit is $15 \mathrm{~mm}$ which corresponds to 3.6 arcminutes in FOV. The slit widths are 100,150, 200, 250, 300 and $350 \mu \mathrm{m}$ which correspond to $1.4,2.1,2.9,3.6,4.3$ and 5 arcseconds in FOV, respectively. The long slits were coated with $\mathrm{Al}$ and $\mathrm{SiO}$ after metal polishing. It is installed in a rotational stage in order to precisely adjust the slit angle. A shutter is attached to the back side of the long slit mount.

In the fiber input, five STU fibers of Polymicro Technologies (Schötz et al., 1998) were connected. Two of them are $300 \mu \mathrm{m}$ in core diameter and 4.2 arcseconds in FOV; another two of them $200 \mu \mathrm{m}$ and 2.8 arcseconds; and one $80 \mu \mathrm{m}, 1.1$ arcseconds. The resolutions of the BOES are measured to $21,000,32,000$ and 80,000 for 300,200 and $80 \mu \mathrm{m}$ fibers. Two fibers of the same size, one for the object and another for the sky background measurement in 200 and $300 \mu \mathrm{m}$ fibers will be used. Whereas, the $80 \mu \mathrm{m}$ fiber will be used for focus checking or high resolution observation programs of bright objects.

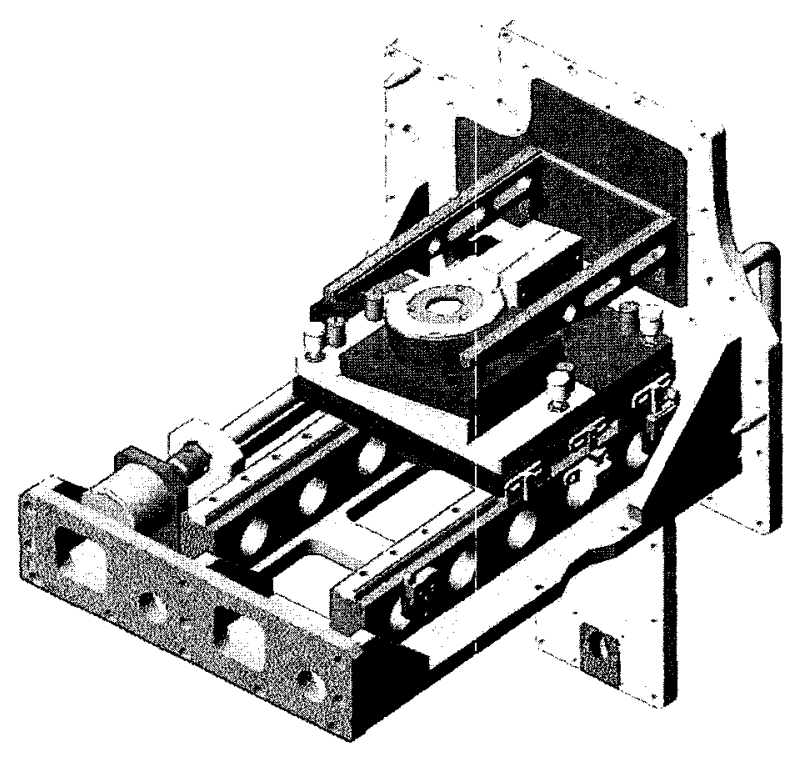

Fig. 2. - Slit Assembly. Slit can be chosen as the Long Slit(left) and Fiber Input V groove (right) are installed on a translation stage. The up side of the fiber input is a polished mirror like as the slit, and the five fibers are inserted as in figure 3.

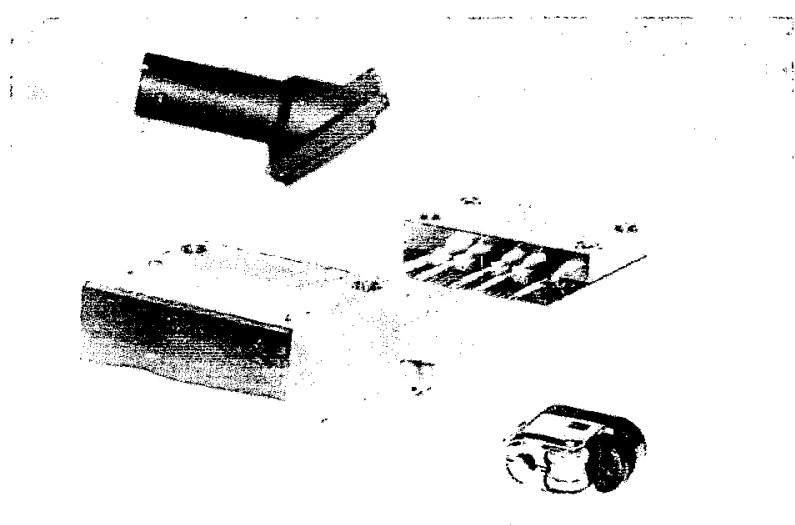

(a)
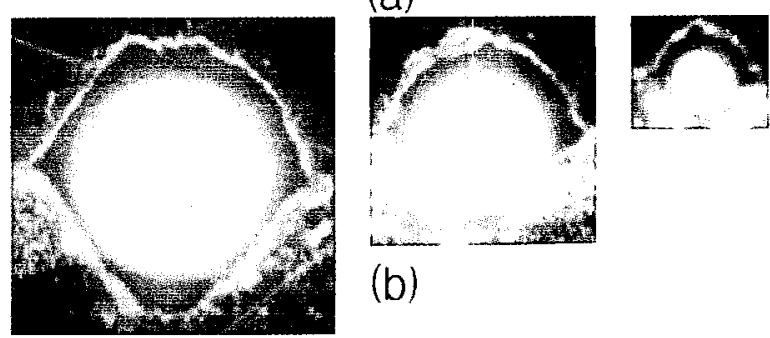

Fig. 3.- (a) Fiber Input. The front surface is polished mirror skewed with 8 degree for the slit monitoring- (b) Enlarged photos of the fiber in this fiber input by a microscope. 300, 200 and $80 \mu \mathrm{m}$ fiber from the left. 


\section{(a) $\mathrm{V}$ groove}

We put these fibers on the $\mathbf{v}$ groove at both ends of the fiber input as shown in Figure 3 and the fiber exit in Figure 4 instead of using the ferrules (Kaufer 1997). The fiber line contacts two points only in the $v$ groove section. Since it does not suffer from any stress, the focal ratio degradation (FRD) due to the stress (Parry 1998, Schmoll et al. 1998) can be reduced.

At the fiber input, two fibers in a given set are seperated by $6 \mathrm{~mm}$ and 1.4 arcminutes in FOV. Each fiber set is apart by $10 \mathrm{~mm}, 2.4$ arcminutes in FOV, from the neighboring one. On the fiber input $\mathrm{v}$ groove, we put a slit cover of $16 \times 22 \mathrm{~mm}$ opening to block the light from the unwanted fibers into the BOES spectrograph room. To fasten the fiber lines on the $\mathrm{v}$ groove, we used a mixed glue, i.e. the epoxy of Loctite E-120HP and fused silica powder of S209 with the mixing weight ratio of epoxy A : epoxy $B$ : glass powder = 1:1:2 (Zajac 2001). While hardening, this mixing epoxy reduce relatively the stress on the fiber, free of heat distortion and shrinking problem. The glass powder makes hardening rapidly within a day. We fixed the fiber line at three points with a small amount of this mixing glue using a toothpick.

In Figure 3 of the fiber input, the front surface is a metal polished mirror with $\mathrm{Al}$ and $\mathrm{SiO} 2$ coated. It is skewed by 8 degrees for slit monitoring. The lid of fiber input $\mathrm{v}$ groove has also five grooves at the exactly same place through the fibers inserted. To minimize the slit monitoring blind area, we made the diameters of the upper lid hole grooves as small as possible, 170, 320 and $420 \mu \mathrm{m}$ in diameter for each of 80,200 and 300 $\mu \mathrm{m}$ fiber. These fiber diameters including the buffer are 102,240 and $370 \mu \mathrm{m}$ respectively with $10 \%$ error. Hence, each fiber blind monitoring diameter becomes $2.4,4.6$ and 6.4 arcseconds. To align the front surfaces of the $\mathrm{v}$ groove and its lid in a plain mirror, four pins were driven during the polishing process of these surfaces. Each fiber is protected in a teflon tube, all of which are again inserted into a metal zebra tube. We put thermal brakes (Fabricant et. al. 1998) between the teflon tubes (see Figure 4).

We did not use a microlens at the fiber input since

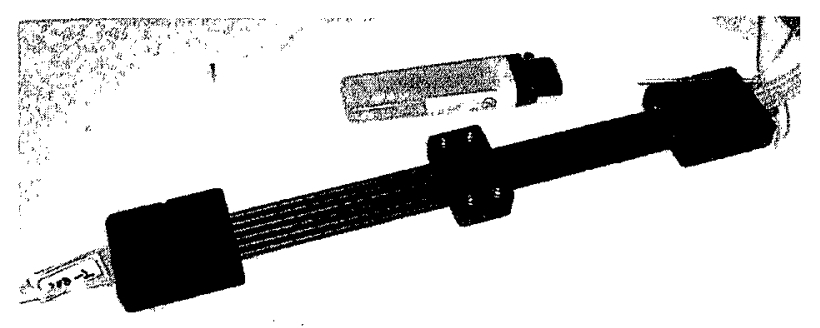

Fig. 4.- Thermal Brake. Fiber do not suffer stress from the thermal expansion of this teflon tube as this tube is disconnected inside of the thermal brake.

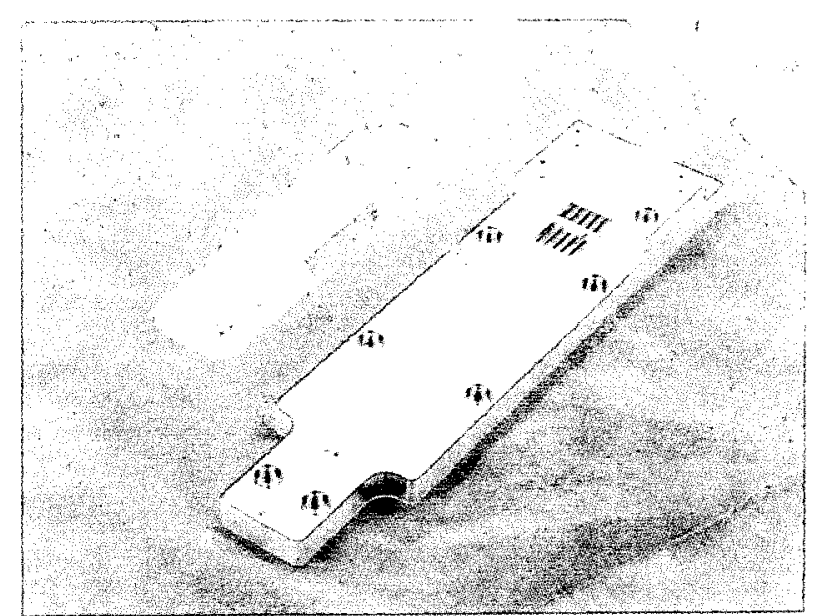

(a)

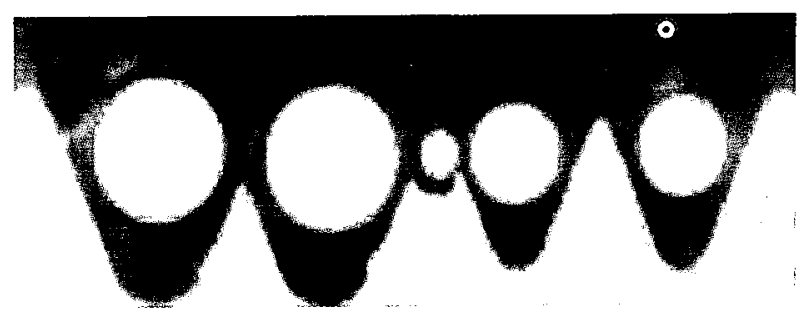

(b)

Fig. 5.- (a) Fiber Exit. (b) Enlarged photo of the fiber exit by a microscpe. The $80 \mu \mathrm{m}$ fiber is at center, and 200 and $300 \mu \mathrm{m}$ fiber sets are at both sides. All these fibers point to the center of the stop $77.1 \mathrm{~mm}$ ahead of this fiber exit.

the small misalignment of the lens (Vogt 1999), the telescope defocusing and bad seeing (Kaufer et al. 1999) would induce much light loss. The light loss due to the FRD from the $\mathrm{f} / 8$ input beam would be $30 \%$ (Avila, Buzzoni and Casse 1998).

In the fiber exit (Figure 5), we put the $80 \mu \mathrm{m}$ fiber at the center, and $200 \mu \mathrm{m}$ and $300 \mu \mathrm{m}$ sets at both sides. We placed one fiber set, apart by $380 \mu \mathrm{m}$ from the other so that their image at the BOES CCD would be apart by $134 \mu \mathrm{m}$ which is a half of the minimum order seperation at the wavelength of $\sim 8,100 \AA$.

The bur in the $\mathrm{v}$ groove which would obstruct the fiber setting and the sharp edge might damage the fiber. Hence, the $v$ groove surface was smoothened with the sandblast, so it removed the bur and chamfered the edge (Zajac 2001). For polishing the fiber, we used wafer mounting wax $\sharp 300$ of Universal Photonics Co. of which hardness is similar to that of glass, and lapping films from 60 to $0.3 \mu \mathrm{m}$. The examination with a microscope showed a good result of fiber polishing. 
(b) Geometrical Efficiency of the Fiber and the Slit

The geometrical efficiency of the light transmission into a fiber and a slit was estimated with respect to seeing and the telescope misguiding. Its result is shown in Figure 6. The dotted, dashed and thread lines in Figure 6 (a) indicate the efficiency for the 80, 200 and 300 $\mu \mathrm{m}$ fibers. In this calculation, $4 \%$ reflectance of the ordinary glass at the fiber input was included. In the Figure 6 (b), the dotted, dashed and thread lines stand for the efficiency for the slits of 100,200 and $300 \mu \mathrm{m}$ width. The seven lines for each curve mean the cases of the seeing size of $1,1.5,2,2.5,3,3.5$ and 4 arcseconds from the top. The figures show that the efficiency depends strongly on the seeing and the guiding error. In the fiber input, the slit monitoring blind diameters are 2.4, 4.6 and 6.4 arcseconds for the 80,200 and 300 $\mu \mathrm{m}$ fibers. To reduce the light loss at the fiber input, the guiding accuracy should be within 0.3 arcseconds, which is the same size of one pixel in the slit monitoring CCD. We revised the telescope control program to obtain this guiding accuracy instead of using track ball as before.

\section{SLIT MONITORING SYSTEM}

The optical layout of the slit monitoring system is shown in Figure 7. Slit image light becomes collimated by $f 260 \mathrm{~mm}$ LAO 238 lens of Melles Griot and focused on a Quantix 57 CCD of Roper Scientific by $\mathrm{f} 160 \mathrm{~mm}$ SH32-2310 lens of Spindler Hoya. Quantix 57 camera employs a back illumination and frame transfer type (ten frames in a second) EEV 57-10 CCD whose maximum efficiency is about $85 \%$ at $600 \mathrm{~nm}$. It is $6.9 \mathrm{x}$ $6.8 \mathrm{~mm}$ CCD, composed of $13 \mu \mathrm{m}$ square $530 \times 526$ pixels. The dark noise is $29 \mathrm{e}^{-} / \mathrm{pixel} / \mathrm{sec}$, while it becomes $18 \mathrm{e}^{-} / \mathrm{pixel} / \mathrm{sec}$ with liquid coolant. The FOV is 2.7 arcminutes ( 0.3 arcseconds per pixel) and RMS spot diameter is $38 \sim 50 \mu \mathrm{m}$ in the field.

We put the LAO 238 lens on the LIMO 30 linear translator for focusing. Some filters such as ND or narrow band pass filter can be placed between the two lenses. We put a $19 \mathrm{~mm}$ diameter stop between the SH32-2310 lens and Quantix camera to block the stray light going into the camera.

\section{CALIBRATION LAMP SYSTEM}

In the CIM, there are two tungsten halogen lamps (THL) of 100 and 10 watts for the flat fielding, and three hollow cathod lamps (HCL) of ThAr and ThNe for the BOES, FeNeArHe for the LSS for wavelength calibration (see Figure 8).

The light from the two THLs goes through the integration sphere of Labsphere US-060-SL. In front of $100 \mathrm{~W}$ THL, red light blocking filters ( $3 \mathrm{~mm} \mathrm{KG3} \mathrm{+} 1$ mm BG39 + 1 mm BG24A) are installed to maintain the color temperature of the two THLs similar to that
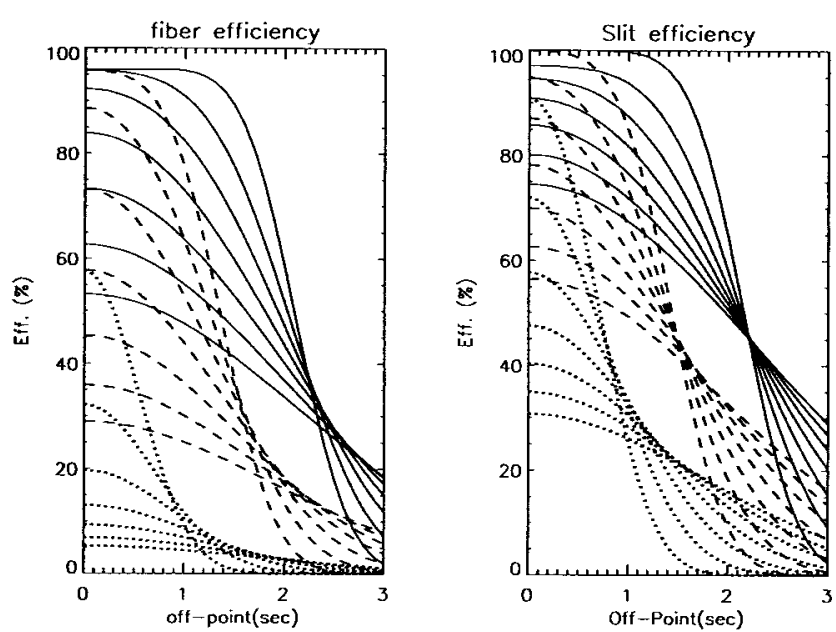

Fig. 6.- Geometrical Light Transmission vs seeing and telescope guiding error in the case of (a) the Fibers and (b) the Slits. Dotted, dashed and thread lines are from the 80, 200 and $300 \mu \mathrm{m}$ fibers in (a) and 100, 200 and $300 \mu \mathrm{m}$ slit widths in (b). Each seven lines are from the seeing sizes of $1.0,1.5,2.0,2.5,3.0,3.5$ and 4.0 arcsec.

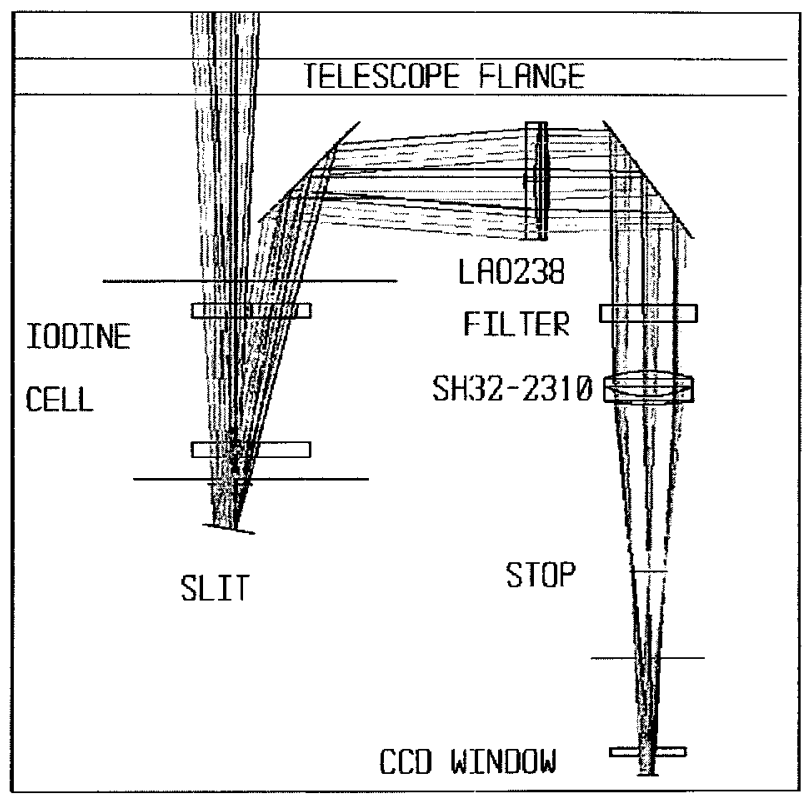

Fig. 7.- The Optical Layout of the Slit Monitoring System. 


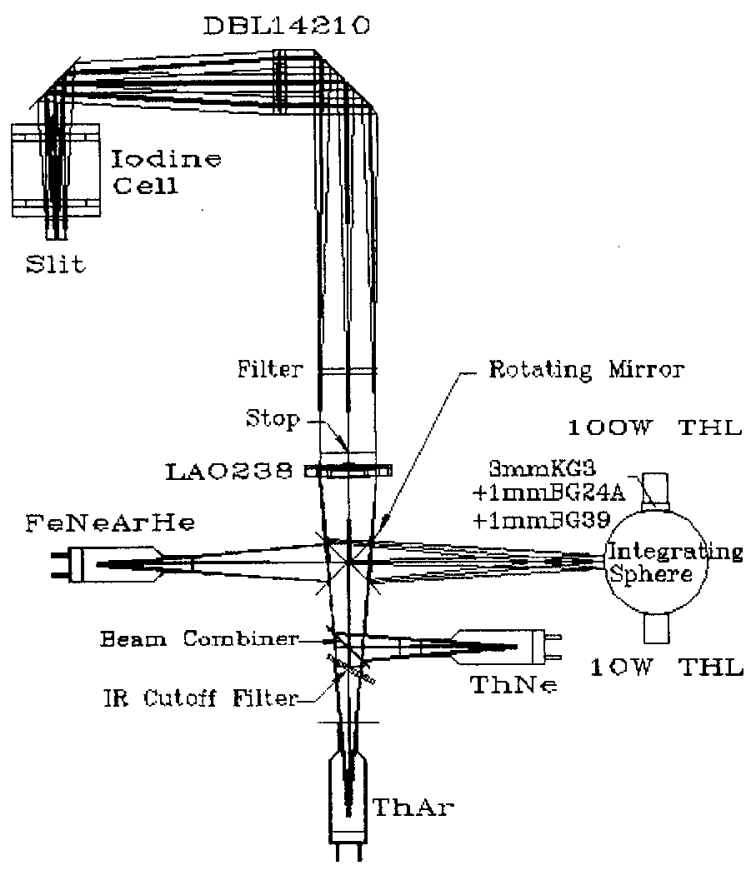

Fig. 8.- The Optical Layout of the Calibration Lamp System.

of star (Kaufer 1997). UV protected window is used in ordianry THL, but we used a pure quartz one because the wavelength range of the BOES is from 360 to 1,050 $\mathrm{nm}$.

For the wavelength calibration of the BOES, ThAr of Photron P858A and ThNe of Photron P858 HCL were adapted. To block the strong light longer than $670 \mathrm{~nm}$ from the Ar lines, we put a IR cutoff filter of Edmund Scientific L54-516 with the size of $50 \times 50 \times 3.3 \mathrm{~mm}$ in front of ThAr lamp. However, since the transmission range of this filter is 400 to $700 \mathrm{~nm}$, IR cutoff filter was tilted with 30 degrees (Kaufer 1997). Two HCL lights -are mixed through beam combiner of Coherent 31-8816 with the size of $50 \times 50 \times 1.2 \mathrm{~mm}$. For the calibration light shorter than $400 \mathrm{~nm}$ and longer than $670 \mathrm{~nm}$, we adapted ThNe lamp in quartz window and the light from this lamp can enter the slit through reflection at the beam combiner. While the ThAr and ThNe lamps are turned on, the rotating mirror is out of the optical path.

The light from the lamps illuminates the stop with f $260 \mathrm{~mm}$ LAO238 lens of Mellis Griot and focuses on the slit with $\mathrm{f} 400 \mathrm{~mm}$ DBL14210 lens of JML. Since the magnification of this calibration lamp optical system is 1.334 , we need $12 \mathrm{~mm}$ light source to illuminate the whole $15 \mathrm{~mm}$ length long slit. For the flat illumination, the light source can irradiate enough broad area because the exit of the integration sphere is relatively large. However, it is impossible for the HCL to illuminate the $15 \mathrm{~mm}$ long slit or two fiber set which is apart by $6 \mathrm{~mm}$ from each other because the illumination area on the slit from the HCL is $\sim 4 \mathrm{~mm}$ with $3 \mathrm{~mm}$ cathod diameter. We cannot use a diffuser such as a ground glass, opal or holographic diffuser, as the light becomes weak, especially shorter wavelength than $370 \mathrm{~nm}$. We could solve these problems by making the FeArNeHe HCL scanning in $\pm 6 \mathrm{~mm}$ for the LSS, and moving the skewed mirror back and forth in front of the slit for the fiber slit.

The light from the calibration sources should have the same exit pupil position and diameter to that from the telescope. In order to obtain this requirement, we calculated the location and the diameter of the primary mirror image by the telescope secondary mirror. The location from the slit is $5,494 \mathrm{~mm}$ and the diameter is $682 \mathrm{~mm}$, so we determine the calibration lamp stop position by tracing the chief ray from the edge, which is $2 \mathrm{~mm}$ from the optical axis, of the illumination at the slit to the direction of primary mirror image center, and stop diameter by the marginal ray. As the LAO238 lens at the backside works as an illuminator to the stop, we put it $10 \mathrm{~mm}$ behind the stop. This lens was chosen by testing the image quality and materials that have good transmission below $400 \mathrm{~nm}$ light and it has a long back focal length enough to give a room for the five calibration lamps.

We design the optical axis of the calibration lamp system $250 \mathrm{~mm}$ apart from the telescope axis. The DBL14210 lens is located $50 \mathrm{~mm}$ apart from the skewed mirror. The $48 \mathrm{~mm}$ diameter stop is $68.72 \mathrm{~mm}$ backward from the cartridge exchange type filter which is $248 \mathrm{~mm}$ from the skewed mirror.

\section{IODINE CELL}

The iodine cell contains iodine gas of $0.001 \mathrm{~atm}$ between two pyrex windows of $75 \mathrm{~mm}$ diameter, 60 $\mathrm{mm}$ clear aperture and $6.2 \mathrm{~mm}$ thickness with antireflection coating whose reflectivity is below $0.5 \%$ between 380 and $620 \mathrm{~nm}$. It is wrapped with nichrome line and insulator. The cell temperature would be maintained at $65 \pm 0.1$ degree in Celsius. The outer dimensions including the insulator is $127 \mathrm{~mm}$ in diameter and $80 \mathrm{~mm}$ in length.

We could get many sharp iodine absorption reference lines overlapping on the stellar continuum between 480 to $620 \mathrm{~nm}$. The accuracy of the radial velocity could be achieved up to $3 \sim 4 \mathrm{~m} / \mathrm{sec}$ (Butler et al. 1996 , Bedding et al. 2001) with a large telescope and 15 $\mathrm{m} / \mathrm{sec}$ (Takeda et al. 2002) with $188 \mathrm{~cm}$ telescope at the Okayama Astrophysical Observatory of Japan.

The cell was installed at $14.1 \mathrm{~mm}$ from the telescope optical axis to the slit monitoring system, so that we could avoid vignetting problem with the small clear aperture of $60 \mathrm{~mm}$ diameter window (see Figure 7). It is parked under the calibration lamp system while not in use. It is controlled by DC motor and mechanical limit switches since it does not require a high positional accuracy. 
Three iodine cells, including the two spare ones, were calibrated with FTS spectrometer at the Kitt Peak National Observatories in the U.S.A.

\section{LONG SLIT SPECTROGRAPH (LSS)}

The medium dispersion spectrograph (MDS), which was used until June of 2002 at the BOAO, was using the $\mathrm{f} / 15$ secondary mirror whereas the CCD imaging observations were peformed with $\mathrm{f} / 8$ secondary mirror. And we experienced some difficulties and inconveniences in light efficiency, wavelength calibration, slit monitoring, autoguiding and maintenance of the telescope (Kim et al. 2001). In addition, many overloaded cables of the MDS made the telescope cable twist system troublesome. To remove these difficulties, we made a new LSS and attached it to the CIM of the BOES. The slit monitoring system and the calibration lamp system of the LSS are sharing with those of the BOES. We reused the grating assembly and the CCD of MDS to the LSS. Therefore newly made parts were only the slit assembly, the collimator and the camera. We adapted the LSS collimator to fit the $\mathrm{f} / 8$ focusing beam which the BOES uses.

The slit of the LSS was described in section II. The collimator is an off-axis parabolic mirror. It has offaxis angle of 14.1 degree, focal length of $635 \mathrm{~mm}$, and diameter of $100 \mathrm{~mm}$ made by SORL. The collimating beam diameter is $79 \mathrm{~mm}$, so the grating assembly of the MDS could be also used in the LSS. We set the angle between the incident collimating beam and the camera to 25 degree.

For the camera, we adapted a commericially manufactured Canon lens since the transmission of the Canon camera with respect to the wavelength was measured the best among the commercial camera lenses (Baudrand and Böhm 1992). We chose $5300 \mathrm{~mm}$ Canon EF f/2.8L USM lens as the camera of the LSS because its transmission data received from Canon Inc. was acceptable. Camera focusing could be done by rotating the camera focus wheel with a stepping motor and a pan belt. And the order sorting filters of BG39, GG495, and RG630 from Andover can be inserted in a filter holder at the end of this camera.

Since we could not obtain the camera optical data from Canon Inc., we tested the ray tracing with the BOES camera data. It seemed to give us satisfactory results.

The plate scale at the CCD is 0.72 arcseconds/px in the spatial direction. The CCD is $24 \mu \mathrm{m}$ square, $1024 \times 1024$ pixel Site CCD. The dispersion and the spectral purity of the LSS are summarized in Table 1. The figure and large alphabet character in the grating name come from the groove number per $\mathrm{mm}$ and blazing wavelength (Kim 1999). The spectral purity was calculated with the slit of $100 \mu \mathrm{m}$ width, 1.43 arcseconds in FOV of which image occupied two pixels in the CCD.

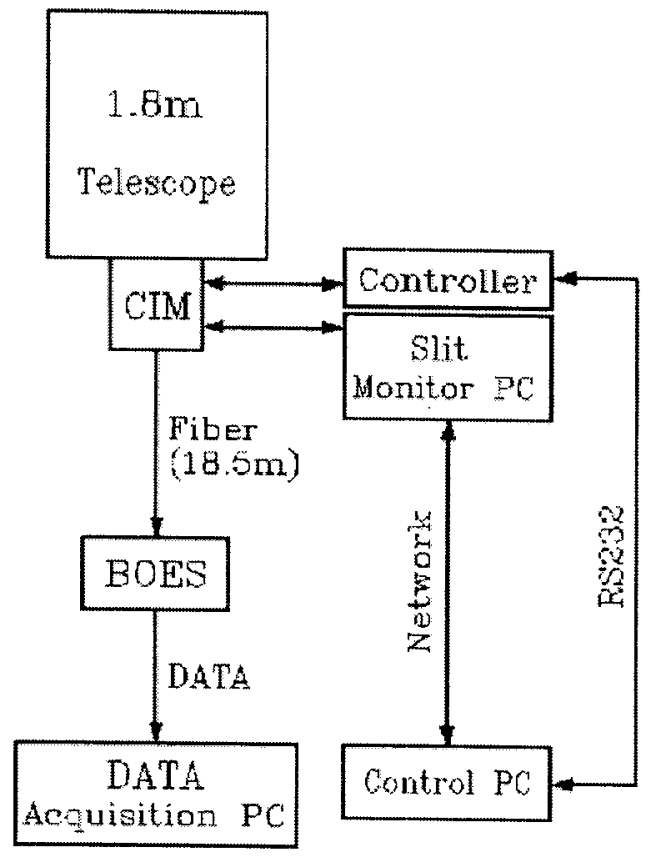

Fig. 9.- The Block Diagram of the BOES

\section{CONTROLLER}

All the control parts of the BOES are confined in the CIM except for the BOES CCD. The controller could adjust the focusing, select the calibration lamp, translate and rotate the mirror and so on. We adapt stepping motors for precise control parts and DC motors for others.

We used STEP 4 board developed at the BOAO for telescope control. STEP4 board can control four axes of stepping motor and give a two byte digital I/O. And the basic control subroutines for the board had already been made earlier. The STEP4 board is connected to $\mathrm{PC}$ by RS232. We made the control software using the CVI compiler of National Instruments.

The block diagram of the BOES is presented in Figure 9. The star light from the fiber input (fiber slit) in the CIM is transmitted to the BOES room in the second floor of the $1.8 \mathrm{~m}$ reflector dome building through $18.5 \mathrm{~m}$ long optical fiber. The BOES room is maintained with temperature variation less than one degree during the night and the humidity below $50 \%$. The Linux based PC in the observing room controls the $2 \mathrm{k}$ $\mathrm{x} 4 \mathrm{k}$ BOES CCD and the shutter. Another control PC in the observing room controls the CIM through RS232 and slit monitoring through network.

\section{CONCLUSION}

We completed the optical design of the CIM by modifying and amending the Vogt's work (1999). The de- 
Table 1. Dispersion and Spectral Purity of the Long Slit Spectrograph (1st order)

\begin{tabular}{cccccccccc}
\hline \hline & unit & $150 \mathrm{~V}$ & $300 \mathrm{~V}$ & $300 \mathrm{I}$ & $500 \mathrm{~V}$ & $600 \mathrm{I}$ & $1200 \mathrm{~V}$ & $1200 \mathrm{R}$ & $1200 \mathrm{I}$ \\
\hline Dispersion & $\AA / \mathrm{mm}$ & 219 & 110 & 111 & 66.5 & 55.5 & 28 & 27 & 27 \\
& $\AA / \mathrm{px}$ & 5.2 & 2.6 & 2.7 & 1.6 & 1.3 & 0.7 & 0.7 & 0.6 \\
Spectral Purity & $\AA$ & 10.4 & 5.2 & 5.4 & 3.2 & 2.6 & 1.3 & 1.3 & 1.3 \\
\hline
\end{tabular}

sign of the mechanical parts, $\mathrm{v}$ grooves and controller, and fiber polishing were done in $\mathrm{BOAO}$. Most mechanical parts were also made by Korean companies except for the commercial optical elements. The preliminary performance test observations so far proved satisfactory results in various respects, but the final report on the BOES is expected early next year after having a full performance test observation.

\section{ACKNOWLEDGEMENTS}

We thank to Dr. Lee, Jong Ung in Chongju Univ., Dr. Kaufer in ESO, Dr. Fabricant and Dr. Zajac in CfA for giving valuable advices and help on our BOES project. The BOES project was carried out with the support of the HAN program from Korean Ministry of Science and Technology. TSY appreciates the support by grant No. R01-2001-000-00026-0 (2001) and No. R01-2001-000-00026-0 (2002) from the Korea Science \& Engineering Foundation.

\section{REFERENCES}

Avila, A., Buzzoni, B., and Casse, M., 1998, SPIE vol.3355, 900, "Fiber Characterization and compact scramblers at ESO"

Baudrand J. and Böhm T., 1992, A\&Ap., 259, 711, "MUSICOS : a fiber-fed spectrograph for multi-site observation",

Bedding T. R., Butler R. P., Kjeldsen H., Baldry I. K., O'Toole S. J., Tinney C. G., Marcy G. W., Kienzle F., Carrier F., 2001, ApJL 549, L105, "Evidence for Solarlike Oscillations in beta HYDRI"

Butler, R. P., Marcy, G. W., Williams, E., McCarthy, C., Dosanjh, P., Vogt, S. S., 1996, PASP, 108, 500, "Attaining Doppler Precision of $3 \mathrm{~m} / \mathrm{sec"}$

Fabricant D. G., Hertz E. N., Szentgyorgyi A. H., Fata R. G., Roll J. B., Zajac J. M., 1998, SPIE vol.3355, 285, "Construction of the Hectospec : 300 optical fiber-fed spectrograph for the converted MMT"

Kaufer, A. 1997, Landessternwarte Heidelberg-Königstuhl, "Final Design Report for the FEROS"

Kaufer, A., Stahl, O., Tubbesing, S., Norregaard, P., Avila, G., Francois, P., Pasquini, A., and Pizzella, A., 1999, Messenger, 95, 8, "Commisioning FEROS, the New High- resolution Spectrograph at La Silla"

Kim, K. M., Jang, J. G., Chun, M. Y., Park, B. G., Hyung, S., Han, I. W., Yoon, "Construction of the BOAO Echelle Spectrograph" (in Korean)
Kim, K. M., Kim, Y. J., Yoon, T. S., 2001, JKAS, 34, 41, "Performance Test of the BOAO Medium Dispersion Spectrograph (MDS)" (in Korean)

Marcy, G. W., Butler R. P., 1992, PASP, 104, 270, "Precision Radial Velocities with an Iodine Absorption Cell"

Parry I. R., 1998, Fiber Optics in Astronomy III, ASP Conference Series, vol. 152, 3, ed. by Arribas S., Mediavilla E., and Watson F., "The Astronomical Uses of Optical Fibers"

Schmoll J., Popow E., and Roth M. M., 1998, Fiber Optics in Astronomy III, ASP Conference Series, vol. 152, 64, ed. by Arribas S., Mediavilla E., and Watson F., "FocalRatio Degradation Optimization for PMAS"

Schötz G. F., Vydra J., Lu G., and Fabricant D., 1998, Fiber Optics in Astronomy III, ASP Conference Series, vol. 152,20 , ed. by Arribas S., Mediavilla E., and Watson F., "New Silica Fiber for Broad-Band Spectroscopy"

Takeda Y., Sato B., Kambe E., Watanabe E., Miyazaki H., Wada S., Ando H., asuda S., Masuda, Izumiura H., Koyano H., Maehara H., Norimoto Y., Okuda T., Shimizu Y., Uraguchi F., Yanagisawa K., Yoshida M., Okuda N., Kawanomoto S., iyama S., 2002, PASJ, 54, 113, "IodineCell Spectroscopy at Okayama Astrophysical Observatory: First Results"

Vogt S. S., 1999, "Phase B Report of the BOES", BOAO

Zajac J. M., 2001, Private Comunication 